\title{
BUTTERFLIES AND MOTHS (INSECTA: LEPIDOPTERA) OF THE LOKRUM ISLAND, SOUTHERN DALMATIA
}

\section{Toni Koren}

Association Hyla, Lipovac I 7, HR-10000 Zagreb, Croatia (e-mail: toni.koren@hhdhyla.hr)

\begin{abstract}
Koren, T.: Butterflies and moths (Insecta: Lepidoptera) of the Lokrum island, southern Dalmatia.
\end{abstract} Nat. Croat., Vol. 29, No. 2, 227-240, 2020, Zagreb.

In 2016 and 2017 a survey of the butterflies and moth fauna of the island of Lokrum, Dubrovnik was carried out. A total of 208 species were recorded, which, together with 15 species from the literature, raised the total number of known species to 223. The results of our survey can be used as a baseline for the study of future changes in the Lepidoptera composition on the island. In comparison with the literature records, eight butterfly species can be regarded as extinct from the island. The most probable reason for extinction is the degradation of the grassland habitats due to the natural succession as well as the introduction of the European Rabbit and Indian Peafowl. Their presence has probably had a tremendously detrimental effect on the native flora and fauna of the island. To conserve the Lepidoptera fauna of the island, and the still remaining biodiversity, immediate eradication of these introduced species is needed.

Key words: Croatia, Adriatic islands, Elafiti, invasive species, distribution

Koren, T.: Danji i noćni leptiri (Insecta: Lepidoptera) otoka Lokruma, južna Dalmacija. Nat. Croat., Vol. 29, No. 2, 227-240, 2020, Zagreb.

U 2016. i 2017. godini provedeno je istraživanje faune danjih i noćnih leptira na otoku Lokrumu kraj Dubrovnika. Zabilježeno je ukupno 208 vrsta, što je zajedno s 15 vrsta navedenih u literaturi povećalo ukupan broj vrsta na 223. Rezultati ovog istraživanja moći će se koristiti kao temelj za praćenje budućih promjena u sastavu leptira na otoku. U usporedbi sa zapisima iz literature, osam vrsta danjih leptira može se smatrati izumrlima na otoku. Najvjerojatniji uzrok tome je degradacija travnjačkih staništa uzrokovana uvođenjem kunića i pauna. Njihova prisutnost vjerojatno ima veliki štetni učinak i na ostalu floru i faunu otoka. Kako bi se doprinijelo zaštiti leptira otoka i očuvala preostala biološka raznolikost, potrebno je u skoroj budućnosti provesti uklanjanje spomenutih vrsta.

Ključne riječi: Hrvatska, jadranski otoci, Elafiti, invazivne vrste, rasprostranjenost

\section{INTRODUCTION}

With 1246 islands, islets and smaller rocks, Croatia has the one of the most rugged coastlines in Europe. From the point of view of area, the islands, islets, and rocks have a total area of $3,259 \mathrm{~km}^{2}$, or about $5 \%$ of the total land area of the country (Duplančić LEDER et al., 2004).

The Adriatic islands are an important part of the country's natural heritage, belonging to the Mediterranean hotspot, one of the six most biodiversity-rich zones on Earth (Médail \& Quézel, 1999). The importance of the Adriatic islands as part of the country's natural heritage has been recognized and several islands have different legal protection statuses. The Brijuni and Kornati islands as well as the western part of Mljet 
island are protected as National Parks while the southern part of Dugi otok island is protected under Telašćica Nature Park. Many islands are also a part of the ecological network Natura 2000.

The exploration of the Lepidoptera fauna of the Adriatic islands started in the beginning of the 20th century and continues even today. Most researchers, however, recorded only butterflies and visited only larger or medium sized islands. The 30 largest Croatian islands account for as much as $92.2 \%$ of the total island area (Nikolić et al., 2008). They are usually better connected and more assessable from the mainland than the smaller islands and islets and thus were more regularly visited in the past. A total of 121 butterfly species have so far been reported for the Croatian islands, comprising more than $60 \%$ of the butterfly fauna of Croatia (VEROVNIK, 2011).

Regarding moths, no systematic overview exists for the Adriatic islands, and the number of species occurring on them is still not known. We can only assume that the moth diversity on the island follows the plant diversity, which is very high on the Adriatic islands. The floristic composition of the Adriatic island is very diverse and the proportion of endemics in the total island flora could reach up to $28.6 \%$ (NiкоLić et al., 2008).

Most of our knowledge about the moth fauna of the Adriatic islands is based on historical records, which are sometimes even more than 100 years old (see GALvAGNI, 1902, 1902; STAUdER, 1921, 1923). A marked exception is the several-decades-long survey of Krk island, the second largest island in the Adriatic Sea, where more than 1600 species have been recorded so far (HABELER, 2008[2003], Gomboc S., unpublished data). Unfortunately, this remains the only island with recently published moth diversity data, while all the other islands still need to be surveyed.

One such un-surveyed island is Lokrum, located about 700 meters from the city of Dubrovnik, southern Dalmatia. With a surface area of $0.694 \mathrm{~km}^{2}$, Lokrum belongs among the smaller Adriatic islands (Duplančić Leder et al., 2004).

It has been protected as a Special Reserve of Forest Vegetation since 1948 (MešTrov, 1989) and is now also a part of the Ecological Network Natura 2000 (HR4000017 Lokrum) due to the presence of eight habitat types important for nature conservation (CRNČEvić et al., 2019). The climate of Lokrum Island is Mediterranean, moderately warm and rainy. The average annual rainfall is $1360 \mathrm{~mm}$ with the most precipitation recorded in November and the least in July.

Almost the entire island is covered with forest vegetation, which contains various stages of development of the plant communities of the Mediterranean, the last stage of which is the community Orno-Quercetum ilicis Horvatić association (IlijANić \& HećIMović, 1989). So far 404 plant species have been recorded on the island, which is relatively high in comparison with the surrounding islands of larger sizes (ILIJANIĆ \& HEćımović, 1989). Grasslands and open habitats are limited to the southwestern part of the island, near the former Benedictine monastery. Vegetation mapping as early as 1959 and 1979 revealed a progressive succession, which was most pronounced on former grasslands and garrigue (Ilijanić \& Hećimović, 1981). In recent times the European Rabbit, Oryctolagus cuniculus (Linnaeus, 1758) and the Indian Peafowl, Pavo cristatus Linnaeus, 1758, were intentionally introduced on the island to raise the attractiveness of the island for visitors. 
The Lokrum island is a popular tourist destination and was also visited by a relatively high number of entomologists in the past (see Đulić \& Durbešić, 1989); neverthess, the Lepidoptera fauna has been poorly studied. Most visitors visited the island only during the day and collected or noted only one or several butterfly species while the moths remain almost completely unknown.

The oldest record of Lepidoptera from Lokrum island is that of DE LA NiCHOLL (1899) who recorded Charaxes jasius (Linnaeus, 1767) on the island. Afterwards, Galvagni (1902) confirmed C. jasius and mentions three additional species: Gonepteryx cleopatra (Linnaeus, 1767), Pieris rapae (Linnaeus, 1758) and Celastrina argiolus (Linnaeus, 1758). GaLvagni (1909) added Lasiommata megera (Linnaeus, 1767) and Endotricha flammealis (Denis \& Schiffermüller, 1775) to the list.

TAвоRsкy (1910) recorded 11 species for the island of which Euchloe ausonia (Hübner, 1804), Gonepteryx rhamni (Linnaeus, 1758), Colias crocea (Geoffroy, 1785), Glaucopsyche alexis (Poda, 1761), Polyommatus icarus (Rottemburg, 1775), Pseudophilotes vicrama (Moore, 1865), Libythea celtis (Laicharting, 1782), Polygonia egea (Cramer, 1775) and Macroglossum stellatarum (Linnaeus, 1758) are mentioned for the first time. STAUder (1921, 1923) only confirmed four previously mentioned butterfly species while SCHAWERDA (1925) mentioned only G. cleopatra.

SCHWingenschuss \& WAgner (1925-1927) recorded four species, of which three were new for the island: Pararge aegeria (Linnaeus, 1758), Limenitis reducta Staudinger, 1901 and Trichoplusia ni (Hübner, 1803). KLIMESCH (1942) reported six Microlepidoptera species from Lokrum island. Burgermeister (1964) includes the observations of six butterfly species from the island, of which three were recorded for the first time: Pieris ergane (Geyer, 1828), Hipparchia fagi (Scopoli, 1763), and Hipparchia statilinus (Hufnagel, 1766). Mladinov (1973) reported seven species from the collections of the Natural History Museum in Zagreb, of which Leptidea sinapis (Linnaeus, 1758), Lycaena phlaeas (Linnaeus, 1761) and Melitaea didyma (Esper, 1778) were not recorded in the past. In total, only 30 Lepidoptera species have been recorded from Lokrum island. This shows that the Lepidoptera fauna of the island was not sufficiently studied and is based only on accidental observations.

The goal of this paper is therefore to present the results of the systematic Lepidoptera survey of Lokrum island near Dubrovnik.

\section{MATERIALS AND METHODS}

Lokrum island was surveyed in 2016 and 2017, four times per year in different vegetation seasons to cover the activity of different Lepidoptera species. During single visits, three to four days were spent on the island in search of Lepidoptera. Dates of visits are as follows: 20-22.4.2016, 23-25.6.2016, 17-19.8.2016, 25-27.10.2016, 28.3.1.4.2017, 8-11.6.2017, 18-21.7.2017, 9-12.10.2017. During each visit to the island seven different localities were surveyed (Tab. 1).

Butterflies and day flying moths were observed or caught with an entomological net during the day. Moths were attracted using standard Lepidoptera equipment. On most localities we used up to six UV light tents. On average, four hours were spent at each locality on each date, depending on the time-of-year and climate conditions.

Where possible, species were identified in the field using standard identification keys and Lepidoptera literature covering also southern Dalmatia (e.g. HAUSMANN \& 
ViIDALePP, 2012; SlAmKA, 2006, 2008, 2013). Questionable material was collected (Permit number: UP/I-612-07/17-48/79), pinned and stored in the Toni Koren private collection in Zagreb. The identification of many species was done by checking the internal genital structures. The genitalia were afterwards placed in micro vials in glycerin and stored with the corresponding specimen.

Tab. 1. Surveyed localities on Lokrum island in 2016 and 2017. Each locality was visited during every visit to the island.

\begin{tabular}{|l|l|c|c|c|}
\hline & Locality & WGS84 S & WGS84 I & $\begin{array}{c}\text { Altitude } \\
\text { (m a.s.l.) }\end{array}$ \\
\hline 1. & Lokrum, maquis near Portoč & 42,626618 & 18,123922 & 40 \\
\hline 2. & Lokrum, surroundings of the monastery & 42,624731 & 18,121364 & 0 \\
\hline 3. & Lokrum, botanical garden & 42,625264 & 18,121176 & 40 \\
\hline 4. & Lokrum, Dead sea, forest edge & 42,623065 & 18,120865 & 0 \\
\hline 5. & Lokrum, Dead sea, maquis and rocky shoreline & 42,621982 & 18,120241 & 0 \\
\hline 6. & Lokrum, maquis near botanical garden & 42,626231 & 18,120237 & 40 \\
\hline 7. & Lokrum, maquis and forest in surrounding of the cape & 42,622009 & 18,119939 & 0 \\
\hline
\end{tabular}

\section{RESULTS AND DISCUSSION}

During our survey, we recorded a total of 208 Lepidoptera species on Lokrum island (Tab. 2) of those 18 butterflies and 190 moth species. The most diverse families were Geometridae and Noctuidae, which corresponds with the general faunistic composition of the fauna in the region (e.g. Schwingenschuss \& WAGNER, 1925-1927). A total of eight butterfly species historically observed on the island were not found during our visits; E. ausonia, L. sinapis, L. phlaeas, G. alexis, P. vicrama, L. celtis, M. didyma, and $H$. statilinus (Taborsky, 1910; Stauder, 1921, 1923; Mladinov, 1973). Another species, H. fagi, has also been mentioned in the literature (Burgermeister, 1964) but this was, most probably, the result of a misidentification of the very similar $H$. syriaca that occurs on the island. Also, seven moth species historically recorded on Lokrum were not confirmed during our survey: Ematheudes punctella (Treitschke, 1833), Eudonia delunella (Stainton, 1849), Pammene oxycedrana (Milličre, 1876), Pammene blockiana (Herrich-Schäffer, 1851), Choreutis nemorana (Hübner, 1799), Mesophleps silacella (Hübner, 1796) (KLIMEscH, 1942) and Trichoplusia ni (Hübner, 1803) (SCHWIngEnschuss \& WAGNER, 1925-1927).

Tab. 2. Recorded Lepidoptera on the Lokrum island during 2016 and 2017.

\begin{tabular}{|c|c|c|c|c|c|c|c|c|}
\hline & \multirow{2}{*}{ List of recorded species } & \multicolumn{7}{|c|}{ Locality number } \\
\hline & & 1 & 2 & 3 & 4 & 5 & 6 & 7 \\
\hline & Papilionidae & & & & & & & \\
\hline \multirow[t]{2}{*}{1.} & Papilio machaon Linnaeus, 1758 & & & & & & + & \\
\hline & Pieridae & & & & & & & \\
\hline 2. & Colias crocea (Geoffroy, 1785) & & & & + & & & \\
\hline 3. & Gonepteryx cleopatra (Linnaeus, 1767 ) & + & & + & + & & + & \\
\hline 4. & Pieris mannii (Mayer, 1851) & + & & & & & & \\
\hline 5. & Pieris brassicae (Linnaeus, 1758) & + & & & + & & & \\
\hline 6. & Pieris ergane (Geyer, 1828) & + & + & + & + & & + & \\
\hline 7. & Pieris rapae (Linnaeus, 1758) & & & & + & + & + & \\
\hline
\end{tabular}




\begin{tabular}{|c|c|c|c|c|c|c|c|c|}
\hline & \multirow{2}{*}{ List of recorded species } & \multicolumn{7}{|c|}{ Locality number } \\
\hline & & 1 & 2 & 3 & 4 & 5 & 6 & 7 \\
\hline & Lycaenidae & & & & & & & \\
\hline 8. & Celastrina argiolus (Linnaeus, 1758) & + & & & + & & & \\
\hline 9. & Favonius quercus (Linnaeus, 1758) & & & & & + & & \\
\hline 10. & Lampides boeticus (Linnaeus, 1767) & & & & & & & + \\
\hline 11. & Leptotes pirithous (Linnaeus, 1767) & & & + & & & & \\
\hline \multirow[t]{2}{*}{12.} & Polyommatus icarus (Rottemburg, 1775) & & & & & & + & \\
\hline & Nymphalidae & & & & & & & \\
\hline 13. & Charaxes jasius (Linnaeus, 1767) & & & & + & & & \\
\hline 14. & Hipparchia syriaca (Staudinger, 1871)* & & & & + & & & \\
\hline 15. & Limenitis reducta Staudinger, 1901 & + & + & & + & + & & + \\
\hline 16. & Pararge aegeria (Linnaeus, 1758) & + & + & & + & & + & + \\
\hline 17. & Vanessa atalanta (Linnaeus, 1758) & + & + & + & + & & & \\
\hline \multirow[t]{2}{*}{18.} & Vanessa cardui (Linnaeus, 1758) & + & & + & + & & & + \\
\hline & Plutellidae & & & & & & & \\
\hline \multirow[t]{2}{*}{19.} & Plutella xylostella (Linnaeus, 1758) & + & & + & + & + & + & \\
\hline & Yponomeutidae & & & & & & & \\
\hline \multirow[t]{2}{*}{20.} & Yponomeuta irrorella (Hübner, 1796) & + & & + & & & & \\
\hline & Chimabachidae & & & & & & & \\
\hline \multirow[t]{2}{*}{21.} & Diurnea fagella (Denis \& Schiffermüller, 1775) & + & & + & & & & \\
\hline & Peleopodidae & & & & & & & \\
\hline \multirow[t]{2}{*}{22.} & Carcina quercana (Fabricius, 1775) & + & & + & & & & \\
\hline & Pterophoridae & & & & & & & \\
\hline 23. & Agdistis bennetii (Curtis, 1833)* & & & + & & & & \\
\hline 24. & Agdistis meridionalis (Zeller, 1847)* & & & + & & & & \\
\hline 25. & Crombrugghia tristis (Zeller, 1841)* & & & & & + & & \\
\hline \multirow[t]{2}{*}{26.} & Emmelina monodactyla (Linnaeus, 1758) & & & + & & & & \\
\hline & Tortricidae & & & & & & & \\
\hline 27. & Piniphila bifasciana (Haworth, 1811) & & & & & & + & \\
\hline 28. & Pseudococcyx tessulatana (Staudinger, 1871) & + & & + & + & + & + & \\
\hline \multirow[t]{2}{*}{29.} & Tortrix viridana Linnaeus, 1758 & & & + & + & & & \\
\hline & Cossidae & & & & & & & \\
\hline 30. & Dyspessa ulula (Borkhausen, 1790) & & & + & & & + & \\
\hline \multirow[t]{2}{*}{31.} & Parahypopta caestrum (Hübner, 1808) & & & & + & & & \\
\hline & Pyralidae & & & & & & & \\
\hline 32. & Denticera divisella (Duponchel, 1842) & & & & & + & & \\
\hline 33. & Dioryctria mendacella (Staudinger, 1859)* & + & & + & & + & & \\
\hline 34. & Dioryctria robiniella (Milličre, 1865$)^{*}$ & & & & & + & & \\
\hline 35. & Ecpyrrhorrhoe diffusalis (Guenée, 1854) & & & + & + & & + & \\
\hline 36. & Endotricha flammealis (Denis \& Schiffermüller, 1775) & + & & + & + & + & & \\
\hline 37. & Hellula undalis (Fabricius, 1781) & & & + & & + & & \\
\hline 38. & Homoeosoma sinuella (Fabricius, 1794) & + & & & & & & \\
\hline 39. & Hypsopygia costalis (Fabricius, 1775) & & & + & + & + & & \\
\hline 40. & Hypsopygia incarnatalis (Zeller, 1847) & + & & & + & & & \\
\hline 41. & Lamoria anella (Denis \& Schiffermüller, 1775) & & & + & & + & & \\
\hline 42. & Loryma egregialis (Herrich-Schäffer, 1838) & & & & + & & & \\
\hline
\end{tabular}




\begin{tabular}{|c|c|c|c|c|c|c|c|c|}
\hline & \multirow{2}{*}{ List of recorded species } & \multicolumn{7}{|c|}{ Locality number } \\
\hline & & 1 & 2 & 3 & 4 & 5 & 6 & 7 \\
\hline 43. & Oncocera semirubella (Scopoli, 1763) & & & + & + & & & \\
\hline 44. & Oxybia transversella (Duponchel, 1836) & + & & + & & + & & \\
\hline 45. & Pyralis farinalis (Linnaeus, 1758) & + & & + & + & & & \\
\hline 46. & Pyralis regalis Denis \& Schiffermüller, 1775 & + & & + & + & + & & \\
\hline 47. & Stemmatophora brunnealis (Treitschke, 1829) & & & & + & & & \\
\hline 48. & Stemmatophora honestalis (Treitschke, 1829) & + & & + & + & + & & \\
\hline \multirow[t]{2}{*}{49.} & Synaphe punctalis (Fabricius, 1775) & & & + & & + & & \\
\hline & Crambidae & & & & & & & \\
\hline 50. & Antigastra catalaunalis (Duponchel, 1833) & & & + & & + & & \\
\hline 51. & Catoptria pinella (Linnaeus, 1758) & + & & & & & & \\
\hline 52. & Cornifrons ulceratalis Lederer, $1858^{*}$ & & & + & & & & \\
\hline 53. & Cydalima perspectalis (Walker, 1859) & + & & + & + & + & & \\
\hline 54. & Diasemiopsis ramburialis (Duponchel, 1834) & & & + & + & & & \\
\hline 55. & Dolicharthria bruguieralis (Duponchel, 1833) & & & + & + & + & & \\
\hline 56. & Dolicharthria punctalis (Denis \& Schiffermüller, 1775) & & & & + & & & \\
\hline 57. & Duponchelia fovealis Zeller, 1847 & & & + & + & & + & \\
\hline 58. & Metasia ophialis (Treitschke, 1829) & + & & + & + & + & & \\
\hline 59. & Metasia rosealis Ragonot, 1895 & & & & & + & & \\
\hline 60. & Nomophila noctuella (Denis \& Schiffermüller, 1775) & + & & + & + & + & + & \\
\hline 61. & Palpita vitrealis (Rossi, 1794) & + & & + & + & & + & \\
\hline 62. & Pleuroptya balteata (Fabricius, 1798) & & & & & + & & \\
\hline 63. & Pleuroptya ruralis (Scopoli, 1763) & & & & & + & & \\
\hline 64. & Pyrausta aurata (Scopoli, 1763) & & & + & & & & \\
\hline 65. & Spoladea recurvalis (Fabricius, 1775 ) & & & + & & & & \\
\hline 66. & Udea ferrugalis (Hübner, 1796) & + & & + & + & & & \\
\hline \multirow[t]{2}{*}{67.} & Uresiphita gilvata (Fabricius, 1794) & + & & + & + & + & & \\
\hline & Drepanidae & & & & & & & \\
\hline 68. & Asphalia ruficollis (Denis \& Schiffermüller, 1775) & & & & + & & & \\
\hline 69. & Polyploca ridens (Fabricius, 1787) & & & & + & & & \\
\hline \multirow[t]{2}{*}{70.} & Watsonalla uncinula (Borkhausen, 1790)* & + & & + & + & + & + & \\
\hline & Lasiocampidae & & & & & & & \\
\hline \multirow[t]{2}{*}{71.} & Dendrolimus pini (Linnaeus, 1758) & + & & + & + & + & + & \\
\hline & Sphingidae & & & & & & & \\
\hline 72. & Daphnis nerii (Linnaeus, 1758 ) & & + & & & & & \\
\hline 73. & Hyles livornica (Esper, 1780) & & & & + & & & \\
\hline \multirow[t]{2}{*}{74.} & Marumba quercus (Denis \& Schiffermüller, 1775) & & & + & + & & & \\
\hline & Geometridae & & & & & & & \\
\hline 75. & Agriopis bajaria (Denis \& Schiffermüller, 1775) & + & & & & & & \\
\hline 76. & Ascotis selenaria (Denis \& Schiffermüller, 1775) & & & + & + & & & \\
\hline 77. & Campaea honoraria (Denis \& Schiffermüller, 1775) & & & + & + & + & + & \\
\hline 78. & Campaea margaritaria (Linnaeus, 1761) & & & + & & & & \\
\hline 79. & Camptogramma bilineata (Linnaeus, 1758) & & & & & + & & \\
\hline 80. & Chemerina caliginearia (Rambur, 1833) & & & + & & & & \\
\hline 81. & Chlorissa cloraria (Hübner, 1813) & & & + & & & + & \\
\hline 82. & Cleora cinctaria (Denis \& Schiffermüller, 1775) & + & & + & + & & & \\
\hline
\end{tabular}




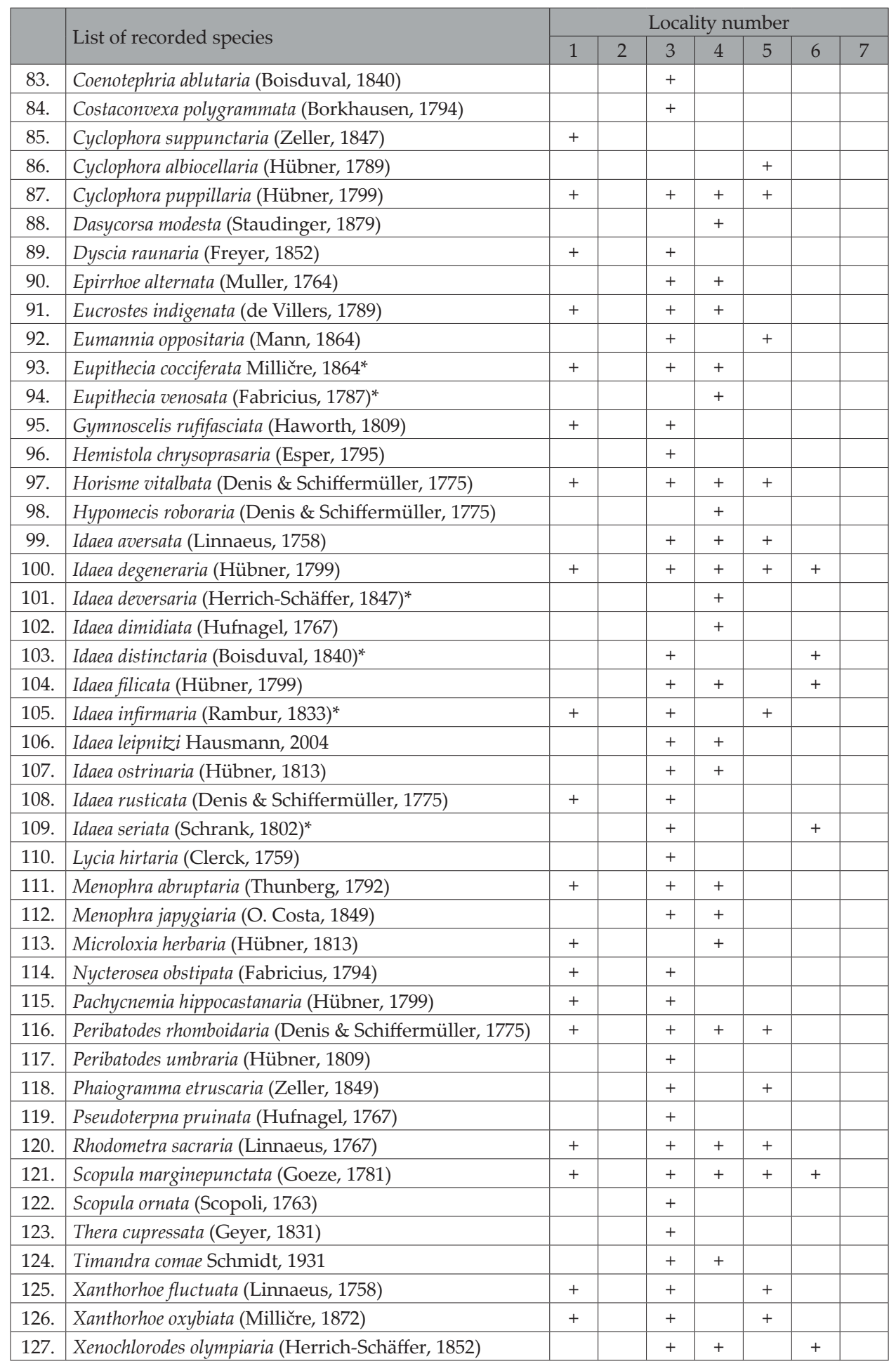




\begin{tabular}{|c|c|c|c|c|c|c|c|c|}
\hline & \multirow{2}{*}{ List of recorded species } & \multicolumn{7}{|c|}{ Locality number } \\
\hline & & 1 & 2 & 3 & 4 & 5 & 6 & 7 \\
\hline & Notodontidae & & & & & & & \\
\hline 128. & Drymonia ruficornis (Hufnagel, 1766) & & & + & & & & \\
\hline 129. & Peridea anceps (Goeze, 1781) & & & + & & & & \\
\hline \multirow[t]{2}{*}{130.} & Thaumetopoea pityocampa (Denis \& Schiffermüller, 1775) & & & + & + & & & \\
\hline & Nolidae & & & & & & & \\
\hline 131. & Nola aerugula (Hübner, 1793) & + & & & & & & \\
\hline 132. & Nola cicatricalis (Treitschke, 1835) & + & & + & + & & + & \\
\hline 133. & Nycteola columbana (Turner, 1925)* & & & & + & & & \\
\hline \multirow[t]{2}{*}{134.} & Nycteola revayana (Scopoli, 1772$)^{*}$ & & & & + & & & \\
\hline & Erebidae & & & & & & & \\
\hline 135. & Atolmis rubricollis (Linnaeus, 1758) & & & + & + & & & \\
\hline 136. & Autophila anaphanes Boursin, 1940 & + & & & & & & \\
\hline 137. & Catephia alchymista (Denis \& Schiffermüller, 1775) & & & + & & & & \\
\hline 138. & Catocala nymphagoga (Esper, 1787) & + & & + & + & + & & \\
\hline 139. & Diaphora luctuosa (Hübner, 1831) & + & & + & + & & & \\
\hline 140. & Dysgonia algira (Linnaeus, 1767 ) & & & + & + & & & \\
\hline 141. & Eilema caniola (Hübner, 1808) & + & & + & + & + & & \\
\hline 142. & Eilema lurideola (Zincken, 1817) & & & & & + & & \\
\hline 143. & Eilema sororcula (Hufnagel, 1766) & & & & + & & & \\
\hline 144. & Eublemma ostrina (Hübner, 1808) & & & & + & & & \\
\hline 145. & Eublemma parva (Hübner, 1808) & + & & + & + & + & & \\
\hline 146. & Eublemma purpurina (Denis \& Schiffermüller, 1775) & & & + & & & & \\
\hline 147. & Eublemma viridula (Guenée, 1841) & + & & + & + & + & & \\
\hline 148. & Grammodes stolida (Fabricius, 1775) & & & & + & & & \\
\hline 149. & Hypena lividalis (Hübner, 1796) & & & & + & + & & \\
\hline 150. & Idia calvaria (Denis \& Schiffermüller, 1775) & + & & & & & & \\
\hline 151. & Lithosia quadra (Linnaeus, 1758) & & & + & + & & & \\
\hline 152. & Lygephila craccae (Denis \& Schiffermüller, 1775) & & & & + & & & \\
\hline 153. & Lygephila procax (Hübner, 1813) & & & & + & & & \\
\hline 154. & Lymantria dispar (Linnaeus, 1758) & + & & + & + & & & \\
\hline 155. & Metachrostis velox (Hübner, 1813) & + & & & & & & \\
\hline 156. & Minucia lunaris (Denis \& Schiffermüller, 1775) & & & & + & & & \\
\hline 157. & Ophiusa tirhaca (Cramer, 1773) & & & + & + & & & \\
\hline 158. & Pechipogo plumigeralis Hübner, 1825 & & & + & & & & \\
\hline 159. & Phragmatobia fuliginosa (Linnaeus, 1758) & & & + & + & & & \\
\hline 160. & Phytometra viridaria (Clerck, 1759) & & & + & + & & & \\
\hline 161. & Zebeeba falsalis (Herrich-Schäffer, 1839) & + & & + & + & + & + & \\
\hline \multirow[t]{2}{*}{162.} & Zekelita antiqualis (Hübner, 1809) & + & & & + & & & \\
\hline & Euteliidae & & & & & & & \\
\hline \multirow[t]{2}{*}{163.} & Eutelia adulatrix (Hübner, 1813) & & & + & + & & & \\
\hline & Noctuidae & & & & & & & \\
\hline 164. & Acronicta euphorbiae (Denis \& Schiffermüller, 1775) & & & + & & & & \\
\hline 165. & Acronicta rumicis (Linnaeus, 1758) & + & & + & + & & & \\
\hline 166. & Aedia funesta (Esper, 1786) & & & & + & & & \\
\hline
\end{tabular}




\begin{tabular}{|c|c|c|c|c|c|c|c|c|}
\hline & \multirow{2}{*}{ List of recorded species } & \multicolumn{7}{|c|}{ Locality number } \\
\hline & & 1 & 2 & 3 & 4 & 5 & 6 & 7 \\
\hline 167. & Agrotis exclamationis (Linnaeus, 1758) & & & + & + & & & \\
\hline 168. & Agrotis ipsilon (Hufnagel, 1766) & + & & + & + & + & & \\
\hline 169. & Agrotis segetum (Denis \& Schiffermüller, 1775) & + & & + & + & + & & \\
\hline 170. & Amphipyra livida (Denis \& Schiffermüller, 1775) & & & & + & & & \\
\hline 171. & Athetis hospes (Freyer, 1831) & & & + & & & & \\
\hline 172. & Callopistria latreillei (Duponchel, 1827) & & & + & + & + & & \\
\hline 173. & Chloantha hyperici (Denis \& Schiffermüller, 1775) & + & & + & + & & & \\
\hline 174. & Conistra rubiginea (Denis \& Schiffermüller, 1775) & + & & + & & & & \\
\hline 175. & Cosmia trapezina (Linnaeus, 1758 ) & & & + & + & & & \\
\hline 176. & Cryphia ochsi (Boursin, 1940)* & + & & + & + & & & \\
\hline 177. & Dryobotodes monochroma (Esper, 1790) & + & & & & + & & \\
\hline 178. & Egira conspicillaris (Linnaeus, 1758) & + & & + & + & & & \\
\hline 179. & Helicoverpa armigera (Hübner, 1808) & & & + & & & & \\
\hline 180. & Heliothis peltigera (Denis \& Schiffermüller, 1775) & & & + & & & & \\
\hline 181. & Leucania loreyi (Duponchel, 1827) & & & + & & & & \\
\hline 182. & Leucania putrescens (Hübner, 1824) & + & & & & & & \\
\hline 183. & Mniotype solieri (Boisduval, 1829) & + & & + & & + & & \\
\hline 184. & Mythimna l-album (Linnaeus, 1767) & + & & + & + & & & \\
\hline 185. & Mythimna unipuncta (Haworth, 1809) & & & + & & & & \\
\hline 186. & Mythimna sicula (Treitschke, 1835$)^{*}$ & + & & + & + & & + & \\
\hline 187. & Noctua comes Hübner, 1813 & + & & + & + & + & & \\
\hline 188. & Noctua fimbriata (Schreber, 1759)* & & & + & + & & & \\
\hline 189. & Noctua janthina Denis \& Schiffermüller, 1775 & + & & + & & + & & \\
\hline 190. & Noctua pronuba (Linnaeus, 1758) & + & & + & + & + & + & \\
\hline 191. & Noctua tirrenica Biebinger, Speidel \& Hanigk, 1983* & & & & + & + & & \\
\hline 192. & Nyctobrya amasina Draudt, $1931^{*}$ & & & + & & & & \\
\hline 193. & Ochropleura leucogaster (Freyer, 1831) & & & + & & & & \\
\hline 194. & Orthosia cerasi (Fabricius, 1775$)^{*}$ & + & & + & + & & & \\
\hline 195. & Orthosia cruda (Denis \& Schiffermüller, 1775) & + & & + & + & & + & \\
\hline 196. & Orthosia miniosa (Denis \& Schiffermüller, 1775) & & & + & & & & \\
\hline 197. & Orthosia gothica (Linnaeus, 1758) & & & & + & & & \\
\hline 198. & Panolis flammea (Denis \& Schiffermüller, 1775) & + & & + & + & & + & \\
\hline 199. & Peridroma saucia (Hübner, 1808) & + & + & + & + & & & \\
\hline 200. & Perigrapha rorida Frivaldszky, 1835 & + & & + & + & & & \\
\hline 201. & Polymixis culoti (Schawerda, 1921) & & & + & & + & & \\
\hline 202. & Polyphaenis sericata (Esper, 1787) & & & + & + & & & \\
\hline 203. & Spodoptera exigua (Hübner, 1808) & + & & + & + & + & & \\
\hline 204. & Xanthia ruticilla (Esper, 1791)* & + & & + & + & & + & \\
\hline 205. & Xestia c-nigrum (Linnaeus, 1758) & + & & & + & & & \\
\hline 206. & Xestia castanea (Esper, 1798) & & & + & & & & \\
\hline 207. & Xestia xanthographa (Denis \& Schiffermüller, 1775) & & & + & & & & \\
\hline 208. & Xylocampa areola (Esper, 1789$)$ & + & & + & + & & + & \\
\hline
\end{tabular}

*Species for which the identification is based on the examination of male or female reproductive organs. 
The data collected during this survey can be used as a baseline for the monitoring of future changes in the number and composition of species of Lepidoptera on the island. Such baseline data are crucial for the management of protected areas; as for Lokrum, recent data about most animal groups are still lacking (CRNČEvić et al., 2019).

This survey greatly enhanced the knowledge of the known Lepidoptera diversity of Lokrum island, which has risen from 30 to 224 known species. With the much larger Krk being the only recently surveyed island in Croatia (HABELER, 2008 [2003]), it is not possible to put our results in perspective in terms of species numbers. Given the size of Lokrum and the limited number of different microhabitats on the island, our results could comprise about $50 \%$ of the potential Lepidoptera fauna on the island.

What should also be taken into consideration is the fact that Lokrum island is located very close to the mainland and a regular intake of the mainland fauna can be expected. This was also observed during our surveys as only on two occasions were single specimens of $P$. icarus seen on the degraded grasslands near the barracks in the central part of the island. This is the same as with $P$. rapae and $P$. brassicae which were observed in low numbers and only occasionally and are possibly not regular residents on the island.

Also, migration waves constituting of a dozen specimens of known migrants like Ochropleura leucogaster and Hyles livornica were observed on the island only once. Thus, it is questionable how many species are permanent inhabitants of the island. Two =such migratory species recorded during this survey, Spoladea recurvalis and Herpetogramma licarsisalis were only recently reported for the first time in Croatia (KoREN \& ZADRAVEC, 2018).

During the identification of the material from Lokrum island, another interesting species, Cornifrons ulceratalis Lederer, 1858 (Fig. 1), was identified. This species occurs throughout northern Africa, the Canary Islands, some of the Mediterranean islands and Iran while in southern Europe it is an occasional migrant (GoATER, 2005). Migration of this species was also recorded in Spain, the Balearic Islands and southern France (DANTART et al., 2009). On Lokrum the species was recorded only once in the Botanical Garden on 29.3.2017 when a single specimen was observed and collected. It was then again observed next year in the vicinity of Dubrovnik, on Mt. Srd, 42,654361N, 18,113417E, 17.3.2018, obs. \& leg. T. Koren, when six specimens were collected. This species has in the past only once been recorded in Croatia, almost a century ago (REBEL, 1926) but has not been included in the recent checklists of the Pyraloidea of Croatia (Gumhalter, 2019a, b) nor was its presence noted for Croatia in the checklist of Pyraloidea of the Balkan peninsula (PLANT \& JAKŠIĆ, 2018). The records from Lokrum island and Srd can be regarded as the first recent observation from Croatia. As for other localities in mainland Europe, this species probably does not reproduce in Croatia but is only an occasional migrant. Recently it was recorded also in Spain and it was categorized as an occasional migrant with low chances of surviving and establishing populations due to the cold winter temperatures and lack of the known host plants used commonly by this species (DANTART et al., 2009).

Aside from the migratory species, there is a large proportion of potential resident species on Lokrum, true Mediterranean elements that are limited to the coastal part of Croatia. Of those Dasycorsa modesta (Staudinger, 1879) (Fig. 2) can be regarded as local and rare, with only a few historical records from the environs of Dubrovnik (ScHwiNGENSCHUSS \& WAGNER, 1925-1927; BURGERMEISTER, 1964). The record from Lokrum island is the first for this species on the Adriatic islands. 


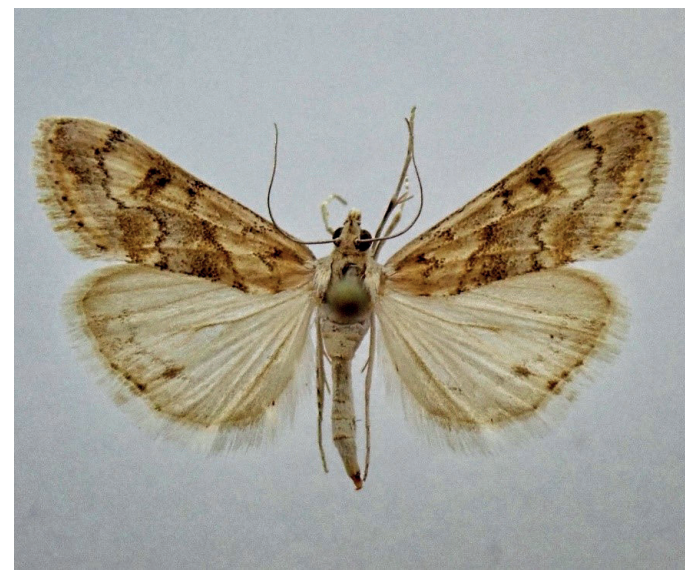

Fig. 1. Cornifrons ulceratalis from Lokrum island (photo by T. Koren).

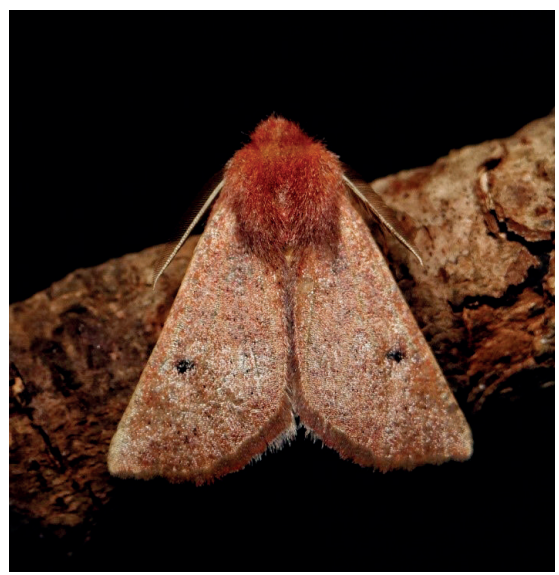

Fig. 2. Dasycorsa modesta from Lokrum island (photo by T. Koren).

Another interesting species recorded on Lokrum is the geometrid Chemerina caliginearia (Rambur, 1833). This Mediterranean species has been recorded in Croatia only near Dubrovnik (Schwingenschuss \& WAgner, 1925-1927). This is probably the first record of this species for the Adriatic islands as well.

Another noteworthy record is Xylocampa areola (Esper, 1789), which has been so far recorded from Lošinj Island (GALVAGNI, 1921) and in the region of the Neretva River in southern Dalmatia (Kučınıć et al., 1998). On Lokrum island the species is generally common in the early spring, regularly visiting lights. It is probably quite widespread in Croatia in the coastal regions of middle and southern Dalmatia (Koren, unpublished data).

This Lepidoptera survey of Lokrum shows the importance and the need for surveying the fauna of small Adriatic islands, most of which have never been systematically surveyed. Due to the lack of surveys, and the lack of moth checklists of the Adriatic islands and Croatia in general, it is very difficult to put this survey into perspective. This is somewhat troubling if the ever-growing tourist pressure on one hand and emigration on the other are considered. The eastern Adriatic coast is one of the most rapidly growing tourist markets within the Mediterranean (PIKELJ \& JuRAčIć, 2013). The faunal composition of the islands is probably influenced by this process, but without any baseline information, which requires extensive financing, such processes are hard to follow.

The forest vegetation of Lokrum island is well preserved, which is also confirmed by the large number of Lepidoptera species whose caterpillars develop on woody plants. However, one of the main threats to the long-term survival of butterflies and moths on the island is lack of nectar sources on the few remaining grassland fragments, as well as in the forest undergrowth. However, the forest undergrowth of these forests is naturally very poor and few plant species were observed in it in the past (ILIJANić \& Hećimović, 1989).

The depletion of the grasslands on Lokrum due to succession was observed several decades ago (Ilijanić \& Hećimović, 1981) and is now complemented by the overwhelming presence of rabbits, peacocks and rats, which were introduced by human activity. Their abundance and effect on the habitats possibly caused the disappearance of several grassland butterfly species from the island. A total of eight butterfly and seven 
moth species historically observed on the island were not found during our visits (Taborsky, 1910; Stauder, 1921, 1923; Klimesch, 1942; Mladinov, 1973). Due to the large sampling effort, we can be almost certain that those species have become extinct on Lokrum island, which is indeed an unique case for the Adriatic islands.

The natural reason for the decline of the aforementioned species is the succession of the grassland, which is ongoing in many places on the island. However, the effect of the introduced species is possibly more prominent, as they directly destroy the nectar sources as well as host plants of the caterpillars. While it is very difficult to remove rats from the islands, it is simpler to do that with the other two species, despite the potentially negative opinion of the general public. The removal of these species could also enable the restoration of the former grassland habitats on the island, which would be beneficial for many insect and plant species. Long term surveys of the Lepidoptera of the Lokrum island would be required to monitor these changes.

\section{ACKNOWLEDGMENTS}

I am grateful to City of Dubrovnik and The Public Institution Lokrum Reserve for financing this survey. I also want to express my gratitude to Ana Štih and Mladen Zadravec for their company and help during field trips. My gratitude goes also to Marija Crnčević and Ozana Domijan for all their help and advice during our stay on the island. I wish to express my gratitude to Rudi Verovnik and an anonymous referee for greatly improving this manuscript.

Received February 13, 2020

\section{REFERENCES}

Burgermeister, F., 1964: Makrolepidopteren aus dem Raume Dubrovnik (Süddalmatien, FVR Jugoslavien). Zeitschrift der Wiener Entomologischen Gesellschaft 49, 137-152.

Crnčević, M., Bratoš Cetinić, A., Pećarević, M., Rajković, Ž. \& Mihaljević, D., 2019: The Contribution of Scientific and Historical Data to the Conservation and Management of the Island of Lokrum Marine Area. Naše more 66, 87-95.

Dantart, J., Stefanescu, C., Avila, A. \& Alarcón, M., 2009: Long-distance windborne dispersal of the moth Cornifrons ulceratalis (Lederer, 1858) (Lepidoptera: Crambidae: Evergestinae) into the northern Mediterranean. European Journal of Entomology 2, 225-229. 10.14411/eje.2009.030.

DE LA Nicholl, B.M., 1899: Butterfly hunting in Dalmatia, Montenegro, Bosnia and Herzegovina. The Entomologist's Record and Journal of Variation 11, 1-8.

Durbešić, P. \& Đulıć, B., 1989: Preliminarni podaci o istraženosti kopnene faune otoka Lokruma, in: Zbornik radova sa Simpozija održanog od 8. - 11.9.1987. u Dubrovniku u organizaciji Sveučilišta u Zagrebu. Hrvatsko ekološko društvo, Dubrovnik, pp. 265-277.

GalvaGni, E., 1902: Beiträge zur Kenntnis der Fauna einiger dalmatischer Inseln. Verhandlungen der k.k. zoologisch-botanischen Gesellschaft 52, 362-380.

Galvagni, E., 1909: Die zoologische Reise des Naturwissenschaftlichen Vereines nach Dalmatien im April 1906. 13. Lepidoptera (Beiträge zur Kenntnis der Lepidopterenfauna der Adriatischen Inseln). Im Selbstverlage des Verfassers. 7, 154-254.

Galvagni, E., 1921: Nachtrag zur Kenntnis der Schmetterlingsfauna Lussins. Verhandlungen der k.k. zoologisch-botanischen Gesellschaft 72, 84-89.

Goater, B., 2005: Evergestinae. In Huemer P. \& Karsholt O. (eds): Microlepidoptera of Europe. Pyraloidea I (Crambidae: Acentropinae, Evergestinae, Heliothelinae, Schoenobiinae, Scopariinae). Part 4. Apollo Books, Stenstrup, pp. 69-107, 208-223, 244-256, pls 2-3.

Gumhalter, D., 2019a: First Checklist of Pyraloid Moths (Lepidoptera: Pyraloidea) in Croatia. Zootaxa 4604(1), 59-102. https://doi.org/10.11646/zootaxa.4604.1.3. 
Gumhalter, D., 2019b: A revised checklist of pyraloid moths (Lepidoptera: Pyraloidea) in Croatia. Natura Croatica 28, 271-288.

KLimesch, J., 1942: Über Microlepidopteren-Ausbeuten aus der Gegend von Zaton bei Gravosa (Süddalmatien). Müchner Entomologischen Gesellschaft 32, 347-399.

Koren, T. \& Zadravec, M., 2018: Three grass moths (Lepidoptera: Crambidae) new to the fauna of Croatia. Natura Croatica 27, 239-242.

Habeler H., 2008[2003]: Die Schmetterlinge der Adria-Insel Krk - eine ökofaunistische Studie. Esperiana - buchreihe zur entomologie, 1-221.

Hausmann, A. \& Viidalepp, J., 2012: Geometrid Moths of Europe, 3. Larentiinae 1. Apollo Books, Stenstrup, 743 pp.

Hećımović, S. \& Ilıjanić, LJ., 1987: Vegetacijske i biljnogeografske značajke dubrovačkog područja s posebnim obzirom na otok Lokrum. Zbornik radova Simpozija Otok Lokrum, Hrvatsko ekološko društvo, Zagreb pp. 139-157.

Ilijanić, Lj. \& S. Hećimović, 1981: Zur Sukzession der mediterranen Vegetation auf der Insel Lokrum bei Dubrovnik. Vegetatio 46, 75-81.

Kučinić, M., JALžıć, B. \& Pelić D., 1998: Xylocampa areola (Esper, 1789), Eurois occulta (Linnaeus, 1758) and Euxoa decora (Denis \& Schiffermüller, 1775), new elements in the noctuid fauna (Insecta: Lepidoptera: Noctuidae) of Croatia. Natura Croatica 7(3), 213-226.

Médail, F. \& QuÉzel, P., 1999: Biodiversity hotspots in the Mediterranean Basin: Setting global conservation priorities. Conservation Biology 13, 1510-1513.

MešTrov, M. (ED.), 1989: Otok Lokrum. Zbornik radova sa Simpozija održanog od 8. - 11.9.1987. u Dubrovniku u organizaciji Sveučilišta u Zagrebu, Ekološke monografije. Hrvatsko ekološko društvo, Zagreb. 485 pp.

Mladinov, L., 1973: Lepidoptera (Rhopalocera) zbirki Hrvatskog narodnog zoološkog muzeja u Zagrebu. Hrvatski narodni zoološki muzej, Zagreb, 175 pp.

Nikolić, T., Oleg, A., Alegro, A., Dobrovi, I., Bogdanovic, S., Liber, Z. \& Rešetnik, I., 2008: Plant species diversity of Adriatic islands: An introductory survey. Plant Biosystems. 142, 435-445.

Pikelj, K. \& M. Juračić, 2013: Eastern Adriatic Coast (EAC): Geomorphology and Coastal Vulnerability of a Karstic Coast. Journal of coastal research 29(4), 944-957.

Plant, C. W. \& P. Jakšić, 2018: A Provisional Checklist and Bibliography of the Pyraloidea of the Balkan Peninsula. Atalanta 49(1-4), 219-63.

Rebel, H., 1926: Ein Beitrag zur Mikrolepidopterenfauna Dalmatiens. Verhandlungen der zoologisch botanischen Gesellschaft in Wien 76, 106-110.

Schawerda, K., 1925. Zur Frage des Vorkommens von Gonepteryx cleopatra in Dalmatien. Zeitschrift des Österreichischen entomologen-vereines 9: 83-85.

Schwingenschuss, L., Wagner, F., 1925: Beitrag zur Macro-Lepidopteen-Fauna Süddalmatiens insbesondere der Umgebung Gravosa's. Zeitschrift des Österreichischen Entomologischen Vereins 10-12, 53-77, 66-71, 78-82, 116-119, 1-3, 9-13, 26-29, 53-54, 67-72, 74-80, 81-86, 45-50, 62-64, 68-72, 73-75.

Slamka, F., 2006: Pyraloidea of Europe (Lepidoptera). Vol. 1. F. Slamka, Bratislava, 138 pp.

Slamka, F., 2008: Pyraloidea of Europe (Lepidoptera). Vol. 2. Crambinae \& Schoenobiinae. F. Slamka, Bratislava, 224 pp.

Slamka F., 2013: Pyraloidea of Europe (Lepidoptera). Vol. 3. Pyraustinae \& Spilomelinae. F. Slamka, Bratislava, $357 \mathrm{pp}$.

StAuder, H., 1921: Die Schmetterlingsfauna der illyro-adriatischen Festland- und Inselzone (Faunula Illyro-Adriatica). Zeitschrift für wissenschaftliche Insektenbiologie Berlin 17, 14-21, 58-64, 83-92, 35-147, 156-176.

Stauder, H., 1923: Die Schmetterlingsfauna der illyro-adriatischen Festland- und Inselzone (Faunula Illyro-Adriatica). Zeitschrift für wissenschaftliche Insektenbiologie Berlin 18, 10-18, 58-68, 106-114, 187-202, 253-267, 317-327.

TÁвоRsку, V., 1910: Eine grössere Sammeltour im slavischen Süden. Entomologische Zeitschrift 23 \& 24 , 224 \& 230; 18-19 \& 24-25.

Verovnik, R., 2011: Butterflies (Lepidoptera: Rhopalocera) of the Croatian islands: an update on published records and new surveys of Pašman and Ugljan. Entomologist's Gazette 62, 251-263. 


\title{
SAŽETAK
}

\section{Danji i noćni leptiri (Insecta: Lepidoptera) otoka Lokruma, južna Dalmacija}

\author{
T. Koren
}

Istraživanja faune danjih i noćnih leptira provode se već stotinjak godina. Fauna danjih leptira većih jadranskih otoka relativno je dobro istražena te je poznato da otoci koji zauzimaju tek 5\% kopnenog dijela Republike Hrvatske sadrže čak $60 \%$ faune danjih leptira. Takvi pregledi su za noćne leptire nepostojeći, a jedini primjer recentno istraživanog otoka je otok Krk, gdje je do sada zabilježeno preko 1600 vrsta leptira. Kako bismo dobili uvid u raznolikost i stanje noćnih leptira na jadranskim otocima, potrebna su novija istraživanja, kako velikih, tako i malih otoka. Otok Lokrum s površinom od svega $0,694 \mathrm{~km}^{2}$ spada u najmanje jadranske otoke. Dio je Elafitskog otočja, a nalazi se u neposrednoj blizini Dubrovnika. U 2016. i 2017. godini provedeno je istraživanje faune danjih i noćnih leptira na otoku Lokrumu. Ukupno je zabilježeno 18 vrsta danjih i 190 vrsta noćnih leptira. Pregledom literature ustanovljeno je da je do sada na otoku Lokrumu zabilježeno svega 30 vrsta, od kojih 15 nismo potvrdili ovim istraživanjem. Time je broj poznatih vrsta danjih leptira na otoku Lokrumu povećan na 223. Na otoku Lokrumu zabilježeno je nekoliko zanimljivih mediteranskih vrsta noćnih leptira, a posebno je značajan nalaz migratorne vrste Cornifrons ulceratalis budući da predstavlja tek drugi nalaz na području Hrvatske. U usporedbi sa zapisima iz literature, osam vrsta danjih leptira i sedam noćnih može se smatrati izumrlima na otoku. Najvjerojatniji uzrok tome je degradacija travnjačkih staništa uzrokovana sukcesijom te uvođenjem kunića i pauna. Njihova prisutnost vjerojatno ima veliki štetni učinak i na ostalu floru i faunu otoka. Rezultati ovog istraživanja moći će se koristiti kao temelj za praćenje budućih promjena u sastavu leptira na otoku, a kako bismo dugoročno zaštitili leptire otoka, kao i ostalu floru i faunu, potrebno je provesti uklanjanje spomenutih vrsta u skoroj budućnosti. 